\section{A BLEAK PICTURE}

Sir, dentistry in India is currently being challenged to maintain its ethical character. We assert this because:

- The examination system used in dental schools is virtually meaningless.

Postgraduate seats are being allotted in private institutes on the basis of capitation fees, not examination performance. (Private institutes are more than 95\% of India's dental schools)

- Most of the dental schools in India do not have an ethics committee. Ethics issues like informed consent and ethical committee clearance are a sham; authors of manuscripts state that such consent and clearance have been obtained, while in reality they have not

- The number of dental institutions has greatly increased, from 120 to 310 in the last ten years, but largely to promote the business of dental education rather than to ensure quality of teaching or graduates' fitness to practise. ${ }^{1}$

The salaries of dental school faculty, unlike salaries for most other professionals, are going down each year; faculty are demoralised and the quality of teaching suffers. Government jobs are rare and are obtained by persons with the greatest influence rather than the best credentials.

There are a few exceptions to this bleak picture. The few public dental institutions are much less corrupt. And there are a few schools, both public and private, that have maintained ethical standards. But, overall, the state of dental education in India engenders feelings of helplessness and hopelessness in us and others in our profession. But we will not give up yet. We call upon the leaders of India's dental profession to have the courage to prevent conditions from deteriorating further and to reverse the damage previously done. For example, an effective and efficient examination system must be developed to maintain the quality of dental graduates. Schools must take ethics standards seriously and create institutional structures to ensure that such standards are observed. (This will require a major culture change in many institutions that, with proper leadership, could occur. Also, those few schools that maintain such standards could be used as examples to the rest.) A minimum pay scale has to be fixed and monitored for dental professionals in private dental schools. Last, consistent with the global competitive standards of the twenty-first century, there is the need for a clear vision of the kind of dentists India should produce. We hope this letter can be helpful to dental and medical professionals in other countries who may be facing similar problems.

A. Singh, B. Purohit Bhopal

1. Sivapathasundharam B. Dental education in India. Indian J Dent Res 2007; 18: 93.

DOI: $10.1038 /$ sj.bdj.2011.208

\section{CONTRADICTING INFORMATION}

Sir, following the publication of my paper Dental technicians: regulation and quality assurance (BDJ 2010; 210: 25-29) I have been informed by the MHRA that dental bleaching trays intended for a cosmetic purpose do not fall within the remit of the Medical Device Regulations and accordingly the new requirement for providing patients with a custom made statement would not apply to such products.

In light of this, dentists who make bleaching trays in their own surgeries do not need to be registered with the MHRA unless they manufacture other custom made medical devices onsite. This therefore contradicts information contained in the 2010 press release: 'Standards updated on commissioning and manufacturing dental appliances' currently posted on the GDC website regarding this matter and which I referenced in my paper.

P. R. H. Newsome Hong Kong DOI: 10.1038/sj.bdj.2011.209 